\title{
Pediatric facial reanimation: An algorithmic approach and systematic review
}

\author{
Paul J. Deramo ${ }^{1}$, Matthew R. Greives ${ }^{2}$, Phuong D. Nguyen ${ }^{2}$ \\ ${ }^{1}$ Division of Plastic Surgery, Department of Surgery and ${ }^{2}$ Division of Pediatric Plastic Surgery, Department of Pediatric Surgery, University of \\ Texas Health Science Center at Houston, McGovern Medical School, Houston, TX, USA
}

Facial palsy has a broad clinical presentation and the effects on psychosocial interaction and facial functions can be devastating. Pediatric facial palsy, in particular, introduces unique familial and technical considerations as anatomy, future growth potential, and patient participation influence treatment planning. Though some etiologies of pediatric facial palsy are self-limiting, congenital and long-standing facial palsies pose difficult challenges that require a combination of surgical, adjunctive, and rehabilitative techniques to achieve facial reanimation. Given the spectrum of ages and symptom severity, as well as the various surgical options available for facial palsy, a tailored approach needs to be developed for each child to restore facial balance and function. Here, we review the etiologies, workup, and treatment of pediatric facial palsy and present our novel algorithmic approach to treatment.

Keywords Facial paralysis / Child / Gracilis muscle / Nerve transfer / Algorithm
Correspondence: Phuong D. Nguyen Division of Pediatric Plastic Surgery, Department of Pediatric Surgery, University of Texas Health Science Center at Houston, McGovern Medical School, 6431 Fannin St, MSB 5.282, Houston, TX 77030, USA

Tel: $+1-713-500-7310$

Fax: +1-713-500-7296

E-mail: Phuong.nguyen@uth.tmc.edu

Received: April 28, 2020 • Revised: June 26, $2020 \bullet$ Accepted: July 15, 2020

pISSN: 2234-6163・ elSSN: 2234-6171 • https://doi.org/10.5999/aps.2020.00710・Arch Plast Surg 2020;47:382-391

\section{BACKGROUND}

Facial muscles are responsible for a wide range of social interactions, emotional expressions, and facial function. Facial palsy, therefore, can be quite debilitating. Eyelid dysfunction and loss of mouth control can lead to visual impairment, problems with speech, salivary control, and communication of emotions. Facial palsy can have profound effects on quality of life including depression and poor self-esteem [1]. While several pediatric societies have different age cutoffs for adulthood, the American Academy of Pediatrics recognizes that pediatric growth and developmental processes generally continue into early adulthoodarbitrarily defined as 21 years of age [2]. The management of facial palsy in children presents distinct challenges as there are additional psychosocial and parental considerations, future growth potential, and anatomical concerns. Depending on the etiology, some pediatric facial palsy may resolve without intervention. However, many palsies persist requiring facial reanimation procedures, adjunct therapies, and rehabilitation in order to restore facial symmetry, balance, and function. Herein, we review causes and treatment of pediatric facial palsy, and propose a novel algorithm to guide decision making.

\section{CAUSES}

The causes of pediatric facial palsy can be generalized into congenital and acquired facial palsy causes. Congenital causes, less than $10 \%$ of all cases, include birth trauma and syndromic facial palsy $[3,4]$. Birth trauma—or obstetric palsy-presents after difficult or prolonged labor and may be associated with forcepsassisted delivery [5]. Newborn infants can have extensive facial bruising, swelling, and other signs of facial trauma. Facial nerve 
compression may occur during delivery or intrauterine positioning and can cause neuropraxia which fortunately usually resolves spontaneously [6]. A relatively common entity is asymmetric crying facies. Though not necessarily a true facial palsy, it is associated with maldevelopment of the depressor anguli oris and may affect up to 1 in 160 children [7]. There are also a number of syndromes associated with congenital facial palsy. Moebius syndrome is the most common congenital syndrome of bilateral facial palsy, estimated at an incidence of 1:50,000. It is characterized by cranial nerve VI and VII palsies leading to symmetric flat facial expressions with associated limb deformities present in roughly $50 \%$ of these children. The deficit of facial musculature results in lower lip incompetence, poor speech and lack of facial animation [8]. CHARGE (coloboma, heart defects, atresia choanae, retardation of growth and/or development, genital and/or urinary abnormalities, and ear abnormalities and/or hearing loss) syndrome, hemifacial microsomia, and 22 q11.2 deletion syndrome are also associated with congenital facial palsy [6].

Acquired facial palsies are responsible for the majority of presentations in the pediatric population [5]. The various causes of pediatric facial palsy are summarized in Table 1. Bell's palsy, or idiopathic facial paralysis, is more commonly seen in adults but still causes up to $40 \%-50 \%$ of all pediatric cases and usually presents unilaterally [3]. It is characterized by sudden onset of loss of facial motion. Though the etiology has not been fully elucidated, viral infections including herpes simplex reactivation are thought to result in facial nerve inflammation in the temporal canal [5]. Upon recognition, steroids should be implemented within 72 hours of onset for maximal effect and prognosis [9]. In over $70 \%$ of patients, symptoms begin to improve within 2 weeks with complete recovery usually by 6 months. Children younger than 8 years old have a higher percentage of full recov-

\section{Table 1. Etiologies of pediatric facial palsy}

\begin{tabular}{|ll|}
\hline Congenital & \multicolumn{1}{c|}{ Acquired } \\
\hline Asymmetric crying facies & Idiopathic \\
Obstetric palsy & Bell's palsy \\
Syndromic & Infectious \\
Moebius syndrome & Otitis media, mastoiditis, Ramsay Hunt syndrome \\
CHARGE syndrome & Varicella, mumps, meningitis, HIV \\
Hemifacial microsomia & Lyme disease \\
22q11.2 Deletion syndrome & Trauma/latrogenic \\
& Neoplastic \\
& Leukemia, rhabdomyosarcoma \\
& Cerebrovascular \\
& Arteriovenous malformation \\
\hline
\end{tabular}

CHARGE, coloboma, heart defects, atresia choanae, retardation of growth and/or development, genital and/or urinary abnormalities, and ear abnormalities and/or hearing loss; HIV, human immunodeficiency virus. ery $[10,11]$. For patients with persistent deficits, surgery may be indicated when no identifiable motor function recovery occurs by 8 months after onset of palsy. Infection in the form of otitis media/mastoiditis, Ramsay Hunt syndrome, and chickenpox may also be responsible for pediatric facial palsy.

Facial palsies are classified by the affected facial nerve anatomic segment. Intracranial etiologies involve all cranial facial nerve segments before the internal acoustic meatus. The intratemporal facial nerve motor segment spans from the internal acoustic meatus to the stylomastoid foramen and branches to supply the stapedius muscle. Beginning at the stylomastoid foramen, the extracranial facial nerve branches into superior and lower trunks which branch between the superficial and deep lobes of the parotid gland before terminating into five branches: temporal, zygomatic, buccal, marginal mandibular, and cervical. Extending medial to the level of the lateral canthus, branches become extremely fine and are difficult to identify and repair.

Intracranial facial palsy etiologies include tumors, infection (e.g., meningitis), vascular insult (stroke), or trauma. Intratemporal causes include tumors, trauma (e.g., temporal bone fracture or penetrating injury), Bell's palsy, and Lyme disease. Extracranial causes include penetrating injury and tumor and may demonstrate a more heterogeneous distribution of clinical findings depending on the location and extent of the injury.

\section{EVALUATION}

\section{Clinical examination}

Treatment planning begins with a thorough history and physical examination to establish timing and chance of recovery without intervention. Pertinent history includes time of onset as well as acuity and progression of symptoms. Though very young children may be unable to fully participate in an examination, at minimum the examiner should note corneal status, obvious facial musculature deficits, oral competence, and facial expression during socialization. When possible, a detailed facial nerve examination may isolate the specific branches of the facial nerve which are affected. A systematic top-down motor exam approach begins by raising eyebrows (temporal branch) and closing the eyes tightly (zygomatic branch) [12]. Eyelid tone, position relative to the cornea, and blink reflex are then assessed to ensure the globe has sufficient protection. To examine the lower face, facial symmetry and tone is assessed at rest and with animation. Comparisons of smile, nasolabial fold, commissure position, and frown of the paralyzed to the uninvolved hemiface are completed to assess specific motor deficits correlating to affected branches. Finally, masseter and temporalis muscle activity confirm normal trigeminal nerve motor function. 


\section{Imaging and electrophysiologic testing}

Computed tomography $(\mathrm{CT})$ and magnetic resonance imaging (MRI) may be obtained during workup of facial palsies. However, these may only be indicated if there is targeted suspicion for lesions or bony trauma affecting the facial nerve function, and are not indicated in most cases of acute onset idiopathic facial palsy [9]. CT reveals intracranial pathology or fractures and MRI may point to facial nerve lesions which can be correlated to specific clinical findings. Two neuroelectrophysiologic studies helpful in facial palsy are electromyography (EMG) and electroneuronography (ENoG). These studies identify the degree of neuronal injury and provide prognostic information regarding spontaneous recovery. Results are most reliable when comparisons are made to unaffected contralateral facial nerves [13]. If examining a patient with new-onset facial palsy, ENoG may be entertained after 72 hours have passed to allow for Wallerian degeneration [13]. Utilizing skin electrodes, evoked muscle action potentials are obtained and compared to the contralateral normal facial nerve. ENoG can be repeated every 3-5 days to determine prognosis of nerve recovery but has little utility beyond 3 weeks [14].

Beyond 3 weeks from the onset of facial palsy, when nerve bodies begin reparative processes which may ultimately lead to complete regeneration, EMG provides more reliable prognosis of nerve recovery $[13,15]$. This modality utilizes needle electrodes and measures evoked motor unit potentials (MUPs) with voluntary facial muscle contraction. Facial nerves that have undergone neuropraxia or axonotmesis-reversible conditionswill gradually demonstrate increasing motor unit action potentials (MUPs) and wave activity before clinically detectable. While neuropraxia resolves quickly, axonotmesis results in Wallerian degeneration and recovery can take several months. During this time, serial EMG performed every 6 weeks with increasing MUPs favors spontaneous nerve recovery. In contrast, neurotmesis, which is irreversible, has EMG findings of fibrillations and absent MUPs persisting over time.

With complete facial palsy less than 6 months duration, serial EMG is recommended to predict nerve recovery and guide treatment options [15]. In contrast, current recommendations for incomplete facial palsy in the setting of Bell's palsy do not require EMG [9]. When nerve recovery is deemed unlikely, the next priority is identification and preservation of remaining viable motor end plates for reinnervation. While there is no universal recommendation in children, reinnervation by $12-18$ months produces optimal recovery [12]. Beyond this, muscles may be irreversibly atrophied. When the predominant symptoms of facial nerve palsy are non-motor (such as hyperacusis or change in taste/salivation), patients often adapt without further treatment.
In the case of congenital or long-standing ( $>18$ months) palsy, physical examination will point to either absent (flaccid paralysis) or marked facial muscle hypoplasia. In either case, the decision to intervene should be informed largely by the age and maturity of the patient. In children under 2 years of age, anticipated growth and technically challenging reconstruction are common reasons reconstructions are delayed until primary school age [16]. In addition, as ultimate facial motion outcomes are predicated on the child's ability to participate with therapy and rehabilitation, it is prudent to wait for children to attain this level of maturation prior to embarking upon reconstruction.

After evaluation and discussion with patient families, the goals of reconstruction are addressed by anatomic zone. In the upper face, eyelid competence is vital to avoid exposure keratopathy. Middle face asymmetry and ptosis should be addressed to improve psychosocial well-being. In the lower face, smile symmetry and oral competence prevent drooling and lead to more accepted social interactions.

\section{TREATMENT}

As discussed earlier, the etiology of facial palsy, duration of symptoms, and laterality guide the treatment algorithm. Establishing the mechanism (congenital vs. acquired) and the timing-early ( $<72$ hours), subacute, and late ( $>18$ months)-helps establish which nerves can be utilized for repair. Static treatment options are helpful for ocular protection and restoring resting symmetry. In contrast, dynamic options allow for functional reanimation and should be the goal whenever possible to allow for facial movements and psychosocial interactions. In adults, factors such as shortened life expectancy, radiation treatment, and severe comorbidities need to be considered before facial reanimation. In contrast, pediatric patients should have robust and sustainable dynamic reconstruction whenever possible.

The potential complications of surgery should be considered when entertaining pediatric facial reanimation. Common complications include hematoma, vascular compromise, paresis, and failed nerve reinnervation [6]. While most complications are minor, flap loss and eventual fibrosis may be potentially devastating and make for more challenging eventual reoperation. Selective muscle resection and scarring from multiple facial dissections may lead to unintended disfigurement. There is some thought that facial reanimation may adversely affect long-term craniofacial growth yet this has not been definitively demonstrated [17]. In contrast, animal studies have suggested the deleterious musculoskeletal growth effects of untreated facial paralysis [18]. As such, we advocate for reanimation options that minimize repeated facial dissection and scar burden. 


\section{Static options}

Though static options are generally reserved for adult patients with limited life expectancy or those who are too frail to undergo more complex reconstructions, they may be helpful in children as adjuncts or as a bridge to dynamic procedures. Static options include slings for lower eyelid and upper lip depression, gold or platinum weight placements to the upper eyelid, and fascia or acellular dermal matrix slings to the midface and oral commissure for improved facial balance. Also, medical adjuncts to improve facial symmetry include botulinum toxin injections to chemically paralyze the normal contralateral lower lip. In addition, contralateral lower lip depressor labii inferioris resection has proven helpful for achieving symmetry in patients with long-standing marginal mandibular nerve palsy [19]. In patients with total orbicularis oculi paralysis, tensor fascia lata slings to the lower eyelid can also provide effective corneal protection [20]. Long-term, however, these static options and adjuncts require multiple revisions as children grow and underscore the importance of robust dynamic reconstructions.

\section{Dynamic options}

Dynamic reconstructive options take advantage of the increased neural plasticity of children with facial palsy to achieve facial balance. The spectrum of options includes nerve repair, neurotization, and muscle replacement in the form of pedicled or free muscle flap reconstruction. Nerve repair is indicated in acute ( $<72$ hours) scenarios such as immediately following tumor extirpation or penetrating trauma. In scenarios where a proximal stump of the facial nerve is unavailable, a babysitter procedure utilizing an alternative nerve source such as the hypoglossal nerve to temporarily innervate the distal targets to prevent muscle atrophy may be indicated [21]. Regional muscle replacement options classically include temporalis turnover and lengthening temporalis myoplasty (LTM), powered by cranial nerve V. Free muscle transfer reconstructive options include free gracilis muscle transfer (FGMT) and latissimus dorsi flaps, powered either by cross-face nerve grafts from the contralateral facial nerve, or the motor nerve to masseter muscle.

\section{Nerve repairs}

For extracranial short nerve gaps $(<1 \mathrm{~cm})$ where the facial nerve can be mobilized, primary repair should be attempted. A tension-free coaptation is critical in order to avoid neuroma or suboptimal reinnervation. If addressed within 72 hours from the onset of palsy, nerve stumps can usually be identified with stimulator. Longer gaps $(>1 \mathrm{~cm})$ usually require nerve conduits or grafts to avoid tensioned coaptation. For gaps $<3 \mathrm{~cm}$, nerve conduits may save time and donor site morbidity. Previous studies have demonstrated $>70 \%$ muscle recovery with nerve conduits for distal facial nerve lesions [22]. For gaps $>3 \mathrm{~cm}$ or when conduits are unavailable, nerve grafts are necessary. The most common donor nerve graft is the sural nerve as it provides consistent anatomy, is easily accessible, has minimal donor site morbidity, and offers long available segments. Alternatively, the great auricular nerve lies within the same surgical field and may negate a separate donor site. Finally, cadaveric allografts are increasingly popular given their widespread availability and lack of donor site.

\section{Nerve transfers and babysitter procedures}

When the proximal facial nerve stump cannot be identified and the distal muscle is viable, neurotization procedures are indicated. This involves utilizing a separate motor nerve source for primary innervation or grafting from a normal contralateral facial nerve branch — the latter usually requiring a "babysitter" procedure. Popularized by Terzis, the babysitter procedure preserves muscle bulk and prevents irreversible atrophy while nerve regeneration ensues over a cross-face nerve graft over a period of 9 to 12 months [21]. The nerve graft, coapted to the buccal branch of contralateral facial nerve, is tunneled across the cheek and buried in the upper buccal sulcus. Identification of the position of the buccal branch may be aided by its consistent anatomy, at the midpoint between the root of the helix to the lateral commissure [23]. A Tinel sign is followed until distal regeneration has occurred and then the patient undergoes a second procedure to divide the babysitter coaptations and coapt the nerve graft. This technique requires an intact contralateral facial nerve as well as two-stage surgery but results in voluntary, emotional facial movements.

In bilateral palsies or when patients do not desire staged surgery, nerve transfers allow for primary innervation of facial musculature. The most common options include fifth-to-seventh cranial nerve and hypoglossal nerve transfer. Spinal accessory or phrenic nerve transfers are legacy operations with significant morbidity and are second-line options [21]. In these techniques, the distal facial nerve serves as a coaptation target. If only distal nerve branches remain, zygomaticus, frontalis, or orbicularis oculi nerve branches may be selected depending on the motor defects. The fifth-to-seventh cranial nerve transfer is a well-described technique with minimal donor site morbidity and relatively consistent anatomy [24]. The motor nerve to the masseter usually has multiple branches with consistent anatomic location at a point $3 \mathrm{~cm}$ from the tragus and $1 \mathrm{~cm}$ below the zygomatic arch [25]. The descending branch can be mobilized and reflected for coaptation with a distal nerve stump. Motor function usually recovers quickly in contrast to the facial nerve, 
though the trigeminal nerve cannot produce emotional muscle function. In the pediatric population, however, neural plasticity and cerebral adaptation may ultimately lead to symmetric facial expressions [26]. The hypoglossal nerve transfer is another popular option in which the ipsilateral hypoglossal nerve is divided and coapated to the distal facial nerve stump. The resulting weakness in tongue movement may affect speech or deglutition and several variations of the technique including end-toside coaptation, split hypoglossal nerve, jump grafts have been described to limit morbidity $[27,28]$.

\section{Regional muscle transfer}

In patients who desire single-stage reconstruction with immediate motor function, or who are not deemed candidates for neurotization and/or free tissue transfer procedures, regional muscle flaps remain a viable reconstructive option. The classic muscle transfers are temporalis muscle-based pedicled flaps or masseter flaps. These are supplied by the trigeminal nerve with resulting motor expression relying on voluntary nerve inputs.

The temporalis muscle flap and masseter muscle flap are used to restore smile asymmetry resulting from facial palsy. Both muscles can be mobilized and rotated on their respective neurovascular supply and appropriately tensioned in a new vector of pull for the oral commissure. The temporalis turnover muscle flap was first described by Gillies in 1934 [29] by elevating the temporalis muscle at its origin and flipping it over the zygomatic arch. The muscle is then anchored to the upper vermilion with the use of fascia lata grafts and, despite obvious bulk, glide easily along the cheek soft tissue. The most potent disadvantages of this technique include donor site hollowing and midfacial widening. As a standalone option, the temporalis turnover flap may provide a symmetric smile though with a strong upward vector of pull which may be balanced with the addition of masseter muscle flap when marginal mandibular nerve function is absent. The anterior portion of the masseter muscle can be transferred to the lower lip medial to the ipsilateral oral commissure in order to balance the upward vector of the temporalis muscle and provide a balanced smile [12]. Unfortunately, the masseter muscle has an unsuitable horizontal vector of pull, making its use as a standalone flap unfavorable for smile reconstruction.

Temporalis muscle can also be lengthened in an orthodromic position as a myoplasty as described by Labbe and Huault [30]. In this case, the temporalis muscle is mobilized from the temporal fossa while retaining its deep temporal arterial branch blood supply. The zygomatic arch is temporarily removed in order to gain exposure to the temporalis insertion at the coronoid. A coronoidectomy is performed and a counterpoint incision is made at the nasolabial fold. Through a series of maneuvers to release interfering fibers of the masseter and pterygoid, the temporalis tendon is then splayed and fixated to the nasolabial fold at the upper lip and commissure. The temporalis muscle (particularly its anterior aspect) is reattached in the temporal fossa, and the zygomatic arch replaced. The muscle's vector of pull utilizes the zygoma as a pulley, and provides a consistent natural appearing smile. As the muscle is deep to the zygomatic arch, this technique avoids the contour deformity and bulk of a temporalis turnover flap. Functional MRI studies have demonstrated cortical plasticity in the centers controlling biting and smiling leading to more voluntary and "spontaneous" effect [31]. The quick restoration of motor function and single-stage non-microsurgical procedure make this an attractive option for pediatric patients with bilateral long-standing facial palsy (Fig. 1, Supplemental Video 1). Furthermore, an inherent advantage is the consistency of the smile vector for bilateral purposes.

\section{Fig. 1. Bilateral lengthening temporalis myoplasty}

Twelve-year-old boy with long-standing bilateral facial nerve palsy after stroke who underwent bilateral lengthening temporalis myoplasty. Preoperative photos at rest (A) and with smiling (B). Four months after surgery and physiotherapy, photos at rest (C) and with smiling (D).
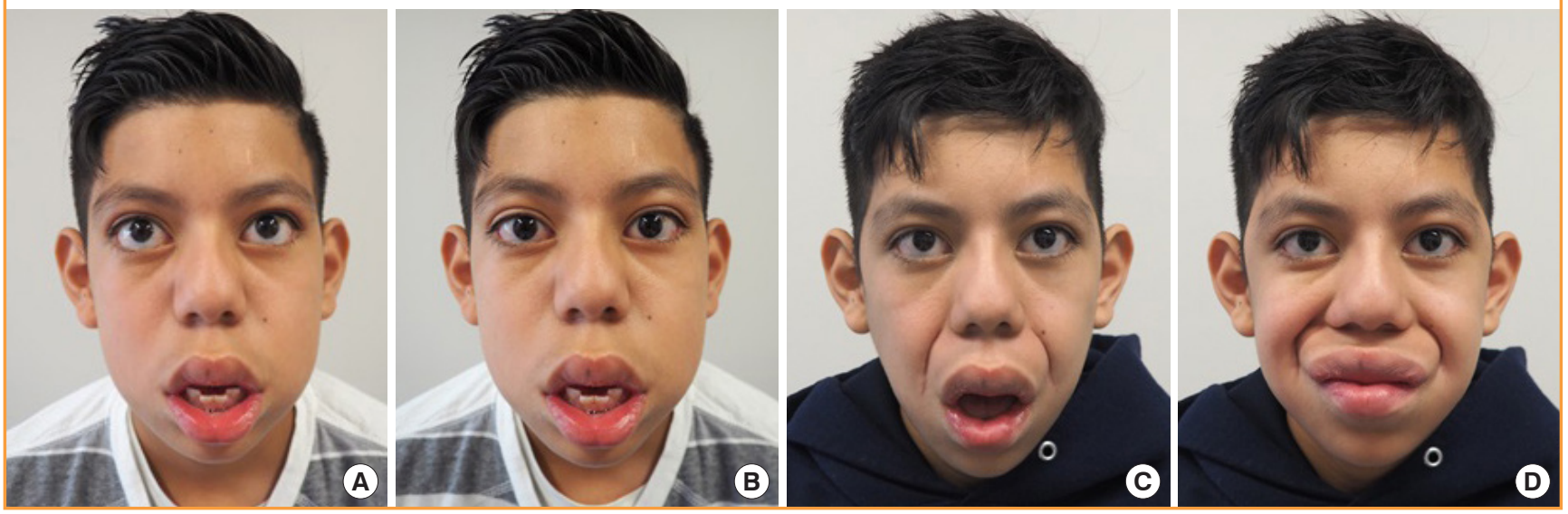


\section{Free tissue transfer}

Highest on the reconstructive ladder, free tissue transfer has arguably become the gold standard in facial reanimation for longstanding facial palsy and gives the most degree of freedom and control of vector of pull with potential for emotional spontaneous expressive motor function.

For unilateral facial palsy, facial reanimation is classically performed in two stages. In the first stage, cross-face nerve grafting with sural nerve coapted to a contralateral intact buccal branch is completed. Then, once the nerve graft has regenerated, microvascular free muscle transfer can be undertaken. While several donor muscles have been described including latissimus and serratus muscles, the gracilis muscle is preferred in most centers given the straightforward harvest, muscle length, and minimal donor site morbidity. The flap is harvested from the contralateral leg intact with the branch of the obturator nerve supplying the muscle. The entire muscle will result in excessive bulk, and as such between one-third and one-half of the muscle width is harvested, taking care to ensure that the vascular pedicle and nerve supply the harvested portion. The face is then exposed through a facelift type incision in a supra-SMAS plane and the flap vessels are anastomosed to recipient vessels, most commonly the facial vessels, utilizing the operative microscope. The muscle is positioned in the correct vector (mimicking the smile vector of the contralateral side) and inset at resting tension. The buried cross-facial nerve graft is identified, and the distal aspect freshened. It is then coapted to the obturator nerve branch using epineural sutures or fibrin sealant in a tension-free manner and the incision closed. For bilateral palsy, contralateral facial reanimation would be completed 3 months later to allow for recovery.
If a single-stage approach is desired, then free muscle transfer is performed in similar fashion, powered by the motor branch to the ipsilateral masseter muscle [8]. The masseteric branch of the trigeminal nerve has a 3-fold increase in axon density compared to cross-facial nerve graft and can supply additional power for gracilis muscle excursion [32]. Unlike the reflexive smile produced from cross-facial nerve grafting, patients must trigger the masseter muscle to produce a smile. Cerebral adaptation can overcome the issues of involuntary facial expression with this reconstruction [33].

More recently, a modification of the two-stage approach to a single-stage procedure has been described that can be employed in children for additional muscle power and voluntary control of facial expression by coapting both the cross-facial nerve graft and the ipsilateral motor branch to the masseter to the obturator nerve [34]. This procedure gives the immediate benefit of power from the masseteric branch while cross-facial nerve branch regeneration slowly provides emotional spontaneous facial expression (Fig. 2, Supplemental Video 2).

\section{Contiguous muscle transfer versus FGMT}

While not as common as FGMT, contiguous muscle transfer procedures are gaining in popularity for treatment of pediatric facial palsy. LTM, for example, has several benefits. The oral commissure remains in a neutral position with the muscle acting as a short-term static sling. Unlike free tissue transfer, the native nerve and vascular connection are maintained so earlier muscle activity can be achieved. Importantly, bilateral contiguous muscle transfer can be performed simultaneously whereas bilateral FGMT is a larger undertaking usually performed at minimum 3 months apart.

\section{Fig. 2. Unilateral dual-innervated free gracilis muscle transfer}

Ten-year-old girl with chronic left facial palsy after resection of brainstem arteriovenous malformation. She underwent left free gracilis muscle transfer, dual innervation with motor nerve to masseter and cross-facial nerve graft. Preoperative photos at rest (A) and with smiling (B). Four months after surgery and physiotherapy, photos at rest (C) and with smiling (D).
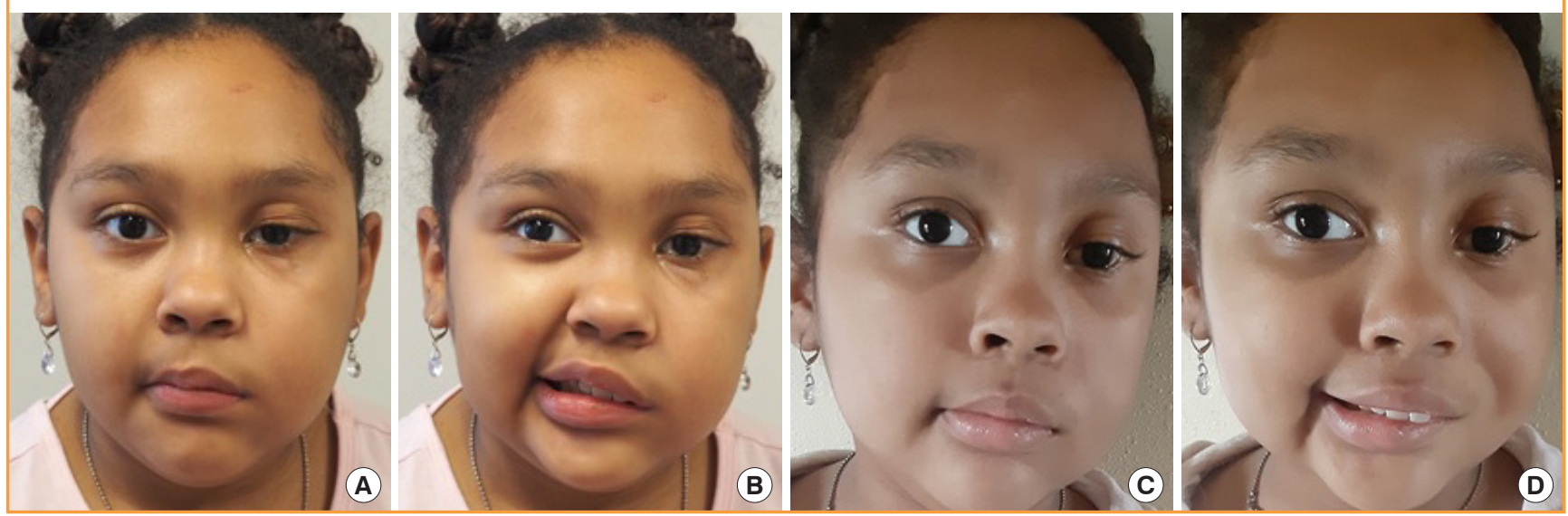
LTM and FGMT with cross-facial nerve grafting remain the two most common dynamic reanimation procedures. Recently, we published the first direct objective comparison of the two procedures in the pediatric population at a single institution [35]. Patients undergoing LTM led to increased early muscle activity and comparable excursion of the oral commissure when compared to those undergoing FGMT. While FGMT with cross-facial nerve grafting would intuitively favor spontaneous smile, the cerebral plasticity of young children may allow for cosmetically and socially acceptable results. Nevertheless, given the complexity of free tissue transfer, longer operative times, and need for staged procedures, patients who desire expeditious recovery with symmetric results may be best served with bilateral LTM [35].

\section{Timing of reconstruction}

Ultimately, four factors drive operative decision-making: etiology of facial palsy, anatomic site of nerve deficit, laterality, and duration of symptoms. Consideration is given to whether the patient is a candidate for prolonged cases utilizing microsurgical free tissue transfer. Fig. 3 summarizes our novel reconstruction algorithm. For congenital or long-standing facial palsy, anatomic site of nerve deficit matters little as the end muscle target may be absent or severely hypoplastic. In unilateral palsies, reconstruction depends on the status of the contralateral facial nerve. If present, cross-facial nerve grafting and free muscle transfer will provide emotional facial expression. Finally, interventions are classified according to their duration of symptoms: immediate

\section{Fig. 3. Algorithmic approach to pediatric facial palsy}

CFNG, cross-facial nerve graft; CN, cranial nerve; EMG, electromyography; ENoG, electroneurography; FGMT, free gracilis muscle transfer; LTM, lengthening temporalis myoplasty.

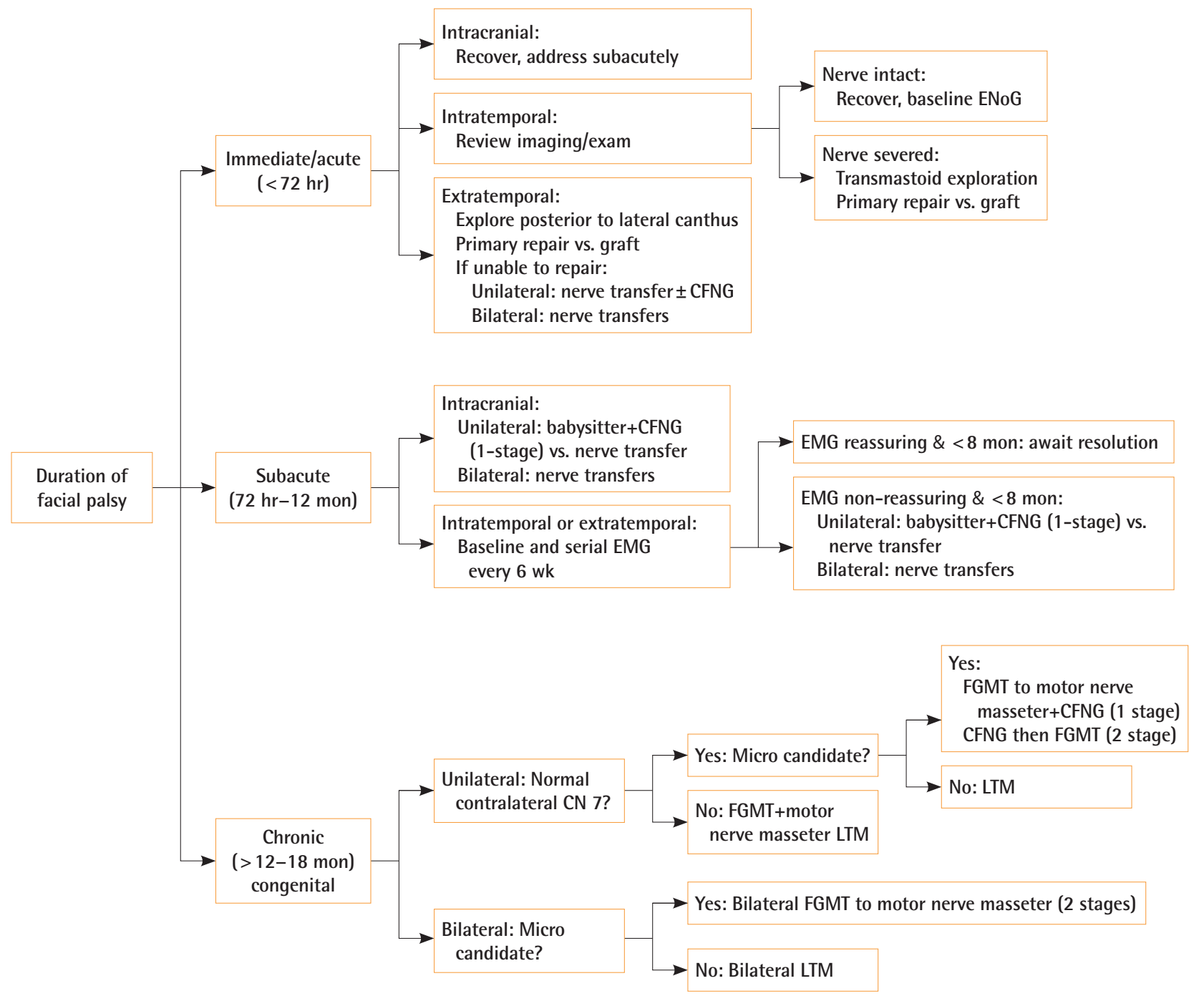


(within 72 hours), subacute ( 72 hours to 12 months) or late (>12 to 18 months).

Immediate interventions are focused on re-establishing nerve conduction. Within 72 hours, a nerve stimulator can be utilized as neurotransmitter stores deplete quickly thereafter making it difficult to stimulate candidate nerve branches during surgery. Primary repair performed as previously described should allow ample time for reinnervation before muscle atrophy. Alternatively, nerve transfer can be performed though commits patient to involuntary motor movements and relies on cerebral plasticity for final function.

Subacute interventions focus on preserving muscle bulk to prevent irreversible atrophy or long-term fibrosis. If history and examination point to transected facial nerve, primary repair should be attempted. Otherwise, baseline EMG is obtained after 3 weeks. When serial EMG and clinical examination reflect spontaneous nerve recovery, expectant management is reasonable. When serial EMG shows no improvement in MUPs and the clinical exam does not progress-particularly after 8 months of palsy-a neurotization procedure is necessary as described previously.

Late interventions or congenital palsies focus on bringing healthy neurotized muscle to the face to restore dynamic facial movements. Regional muscle transfer or free muscle transfer are both tenable options and treatment depends in large part on microsurgical candidacy and laterality of deficits. If unilateral and the patient is a microsurgical candidate, we prefer a dual-innervated (ipsilateral motor branch to the masseter and cross-facial nerve graft) FGMT approach which allows for early involuntary muscle movement and eventual emotional spontaneous expression. The classic two-stage FGMT approach is also utilized in our institution for unilateral facial palsy, and determination of utilizing this method versus the single-stage dual innervation is a joint decision with the patient and their family based on individualized priorities and clinical situation. If bilateral and the patient is a microsurgical candidate, we perform two-stage FGMT utilizing motor branch of the masseter with at least 3 months between stages versus single-stage bilateral LTM.

As discussed, patient age guides when to offer facial reanimation. Though durable facial reanimation has been achieved in toddlers, delaying surgeries until school age (typically 5-6 years old) allows time for children to become mindful and more active participants in their operative care and therapy. Larger recipient vessel size allows for more reliable free tissue transfer. However, once reaching school age, children benefit long-term from dynamic operations whereas static options should be reserved for those who in early adulthood who have completed most of their growth. Table 2 summarizes reconstructive tech-
Table 2. Pediatric facial palsy interventions by age

\begin{tabular}{|c|c|c|}
\hline Age & Procedures & Recommendation \\
\hline \multirow[t]{5}{*}{$2-5 \mathrm{yr}$} & Conservative management & \\
\hline & Static (Botox/sling) & $\begin{array}{l}\text { Avoid, requires frequent revision due to } \\
\text { growth }\end{array}$ \\
\hline & Nerve procedures & $\begin{array}{l}\text { Perform nerve repair or transfers when } \\
\text { feasible (see Fig. 3) }\end{array}$ \\
\hline & Regional muscle transfer & Avoid, limited participation in therapy \\
\hline & Free tissue transfer & $\begin{array}{l}\text { Avoid, limited participation in therapy; } \\
\text { technically challenging }\end{array}$ \\
\hline \multirow[t]{5}{*}{$6-21 \mathrm{yr}$} & \multicolumn{2}{|l|}{ Operative intervention } \\
\hline & Static (Botox/sling) & $\begin{array}{l}\text { Perform once most growth completed to } \\
\text { limit revisions }\end{array}$ \\
\hline & Nerve procedures & $\begin{array}{l}\text { Perform nerve repair or transfers when } \\
\text { feasible (see Fig. 3) }\end{array}$ \\
\hline & $\begin{array}{l}\text { Regional muscle transfer, } \\
\text { (i.e., LTM) }\end{array}$ & $\begin{array}{l}\text { Perform when microsurgery } \\
\text { contraindicated, quick recovery desired, } \\
\text { or bilateral disease }\end{array}$ \\
\hline & Free tissue transfer & $\begin{array}{l}\text { Perform FGMT as 2-stage (CFNG, then } \\
\text { FGMT) versus 1-stage (CFNG+motor } \\
\text { nerve to masseter+FGMT) }\end{array}$ \\
\hline \multicolumn{3}{|c|}{$\begin{array}{l}\text { LTM, lengthening temporalis myoplasty; FGMT, free gracilis muscle transfer; } \\
\text { CFNG cross-facial nerve araft. }\end{array}$} \\
\hline
\end{tabular}

niques and recommendations by age.

After facial reanimation, we enroll our patients in physiotherapy with guided exercises to specific muscle groups and neural inputs utilized for reconstruction. Our physiotherapy protocol begins 3 weeks postoperatively. Specific exercises are tailored to muscle group and neural inputs powering the reconstruction. For patients undergoing LTM, we follow Martin's previously published "mirror-effect" protocol [36]. Muscle exercises are commenced preoperatively to give sufficient autonomy to the temporalis muscle. In those undergoing regional muscle transfer, earlier muscle activity allows for faster time to physiotherapy and shorter rehabilitation. There is a dearth of literature comparing patient-reported outcomes and smile spontaneity between regional muscle and free muscle transfer procedures for facial reanimation.

\section{CONCLUSIONS}

Pediatric facial palsy can have profoundly debilitating effects on emotional expression, psychosocial interaction, and complex facial actions. Regardless of etiology and whether surgical intervention is warranted, children with facial palsy have decreased quality of life and often deleterious mental/emotional effects. The management of facial palsy has evolved in the past century with the perfection of microsurgical free tissue transfer and improved understanding of neural surgery. Though static options for reconstruction are acceptable in some instances, children are capable of cerebral plasticity and benefit from the durable re- 
sults provided by dynamic facial reanimation. Our algorithmic approach to pediatric facial reanimation considers the etiology, duration, and laterality of symptoms as well as candidacy for prolonged microvascular surgery. While further research should focus on functional, aesthetic, and patient-reported outcomes, institution of an algorithm simplifies the evaluation and effectively guides treatment of pediatric facial palsy.

\section{NOTES}

\section{Conflict of interest}

No potential conflict of interest relevant to this article was reported.

\section{Patient consent}

The parents of the patients provided written informed consent for the publication and the use of their images.

\section{Author contribution}

Conceptualization: MR Greives, PD Nguyen. Data curation: PJ Deramo. Methodology: PJ Deramo, PD Nguyen. Project administration: PJ Deramo, PD Nguyen. Writing - original draft: PJ Deramo. Writing - review \& editing: PJ Deramo, MR Greives, PD Nguyen.

\section{ORCID}

Paul J. Deramo ～https://orcid.org/0000-0002-1123-3761

Matthew R. Greives https://orcid.org/0000-0003-4602-5054

Phuong D. Nguyen https://orcid.org/0000-0002-2116-902X

\section{Supplementary material}

Supplemental Video 1. Intraoperative video of bilateral lengthening temporalis myoplasty. Supplemental data can be found at: https://doi.org/10.5999/aps.2020.00710.v001.

Supplemental Video 2. Intraoperative video of unilateral dualinnervated free gracilis muscle transfer. Supplemental data can be found at: https://doi.org/10.5999/aps.2020.00710.v002.

\section{REFERENCES}

1. Dey JK, Ishii M, Boahene KD, et al. Changing perception: facial reanimation surgery improves attractiveness and decreases negative facial perception. Laryngoscope 2014;124: 84-90.

2. Hardin AP, Hackell JM; Committee on Practice and Ambulatory Medicine. Age limit of pediatrics. Pediatrics 2017; 140:e20172151.
3. Manning JJ, Adour KK. Facial paralysis in children. Pediatrics 1972;49:102-9.

4. May M, Fria TJ, Blumenthal F, et al. Facial paralysis in children: differential diagnosis. Otolaryngol Head Neck Surg 1981;89:841-8.

5. Pavlou E, Gkampeta A, Arampatzi M. Facial nerve palsy in childhood. Brain Dev 2011;33:644-50.

6. Banks CA, Hadlock TA. Pediatric facial nerve rehabilitation. Facial Plast Surg Clin North Am 2014;22:487-502.

7. Shapira M, Borochowitz ZU. Asymmetric crying facies. NeoReviews 2009; 10:e502-9.

8. Zuker RM, Goldberg CS, Manktelow RT. Facial animation in children with Möbius syndrome after segmental gracilis muscle transplant. Plast Reconstr Surg 2000;106:1-9.

9. Baugh RF, Basura GJ, Ishii LE, et al. Clinical practice guideline: Bell's palsy. Otolaryngol Head Neck Surg 2013;149(3 Suppl):S1-27.

10. Chen WX, Wong V. Prognosis of Bell's palsy in children: analysis of 29 cases. Brain Dev 2005;27:504-8.

11. Lee Y, SooYoon H, Yeo SG, et al. Factors associated with fast recovery of Bell palsy in children. J Child Neurol 2020;35: 71-6.

12. Garcia RM, Hadlock TA, Klebuc MJ, et al. Contemporary solutions for the treatment of facial nerve paralysis. Plast Reconstr Surg 2015;135:1025e-1046e.

13. Chu EA, Byrne PJ. Treatment considerations in facial paralysis. Facial Plast Surg 2008;24:164-9.

14. Brown S, Isaacson B, Kutz W, et al. Facial nerve trauma: clinical evaluation and management strategies. Plast Reconstr Surg 2019;143:1498-512.

15. Sittel C, Stennert E. Prognostic value of electromyography in acute peripheral facial nerve palsy. Otol Neurotol 2001; 22:100-4.

16. Hughes CA, Harley EH, Milmoe G, et al. Birth trauma in the head and neck. Arch Otolaryngol Head Neck Surg 1999;125:193-9.

17. Terzis JK, Olivares FS. Long-term outcomes of free muscle transfer for smile restoration in children. Plast Reconstr Surg 2009; 123:543-55.

18. Sinsel NK, Guelinckx PJ. Effect of unilateral partial facial paralysis on periosteal growth at the muscle-bone interface of facial muscles and facial bones. Plast Reconstr Surg 2003; 111:1432-45.

19. Hussain G, Manktelow RT, Tomat LR. Depressor labii inferioris resection: an effective treatment for marginal mandibular nerve paralysis. Br J Plast Surg 2004;57:502-10.

20. Sendul SY, Cagatay HH, Dirim B, et al. Effectiveness of the lower eyelid suspension using fascia lata graft for the treat- 
ment of lagophthalmos due to facial paralysis. Biomed Res Int 2015;2015:759793.

21. Terzis JK, Tzafetta K. The "babysitter" procedure: minihypoglossal to facial nerve transfer and cross-facial nerve grafting. Plast Reconstr Surg 2009; 123:865-76.

22. Navissano M, Malan F, Carnino R, et al. Neurotube for facial nerve repair. Microsurgery 2005;25:268-71.

23. Dorafshar AH, Borsuk DE, Bojovic B, et al. Surface anatomy of the middle division of the facial nerve: Zuker's point. Plast Reconstr Surg 2013;131:253-7.

24. Klebuc MJ. Facial reanimation using the masseter-to-facial nerve transfer. Plast Reconstr Surg 2011;127:1909-15.

25. Borschel GH, Kawamura DH, Kasukurthi R, et al. The motor nerve to the masseter muscle: an anatomic and histomorphometric study to facilitate its use in facial reanimation. J Plast Reconstr Aesthet Surg 2012;65:363-6.

26. Lifchez SD, Matloub HS, Gosain AK. Cortical adaptation to restoration of smiling after free muscle transfer innervated by the nerve to the masseter. Plast Reconstr Surg 2005; 115 : 1472-82.

27. Venail F, Sabatier P, Mondain M, et al. Outcomes and complications of direct end-to-side facial-hypoglossal nerve anastomosis according to the modified May technique. J Neurosurg 2009; 110:786-91.

28. May M, Sobol SM, Mester SJ. Hypoglossal-facial nerve interpositional-jump graft for facial reanimation without tongue atrophy. Otolaryngol Head Neck Surg 1991;104:818-25.
29. Gillies H. Experiences with fascia lata grafts in the operative treatment of facial paralysis (section of otology and section of laryngology). Proc R Soc Med 1934;27:1372-82.

30. Labbe D, Huault M. Lengthening temporalis myoplasty and lip reanimation. Plast Reconstr Surg 2000;105:1289-98.

31. Garmi R, Labbe D, Coskun O, et al. Lengthening temporalis myoplasty and brain plasticity: a functional magnetic resonance imaging study. Ann Chir Plast Esthet 2013;58:271-6.

32. Snyder-Warwick AK, Fattah AY, Zive L, et al. The degree of facial movement following microvascular muscle transfer in pediatric facial reanimation depends on donor motor nerve axonal density. Plast Reconstr Surg 2015;135:370e-381e.

33. Buendia J, Loayza FR, Luis EO, et al. Functional and anatomical basis for brain plasticity in facial palsy rehabilitation using the masseteric nerve. J Plast Reconstr Aesthet Surg 2016;69:417-26.

34. Biglioli F, Colombo V, Tarabbia F, et al. Double innervation in free-flap surgery for long-standing facial paralysis. J Plast Reconstr Aesthet Surg 2012;65:1343-9.

35. Nguyen PD, Faschan KS, Mazzaferro DM, et al. Comparison of lengthening temporalis myoplasty and free-gracilis muscle transfer for facial reanimation in children. J Craniofac Surg 2020;31:85-90.

36. Blanchin T, Martin F, Labbe D. Lengthening temporalis myoplasty: a new approach to facial rehabilitation with the "mirror-effect” method. Ann Chir Plast Esthet 2013;58:632-7. 